\section{Biotic, Edaphic, and Cultural Factors Associated with Strawberry Black Root Rot in New York}

\author{
Katherine B. Wing and Marvin P. Pritts \\ Department of Fruit and Vegetable Science, Cornell University, Ithaca, \\ NY 14853 \\ Wayne F. Wilcox \\ Department of Plant Pathology, Cornell University, New York Agricultural \\ Experiment Station, Geneva, NY 14456
}

Additional index words. Fragaria $\times$ ananassa, Rhizoctonia, Pythium, Pratylenchus, metalaxyl, terbacil, soil compaction

\begin{abstract}
Blackening and decay of roots in association with plant stunting are common in perennial strawberry (Fragaria $\times$ ananassa Duch.) plantings worldwide; this syndrome is commonly referred to as black root rot (BRR), although its causal agent(s) are not well characterized. We conducted a New York field survey that measured many physical and cultural factors in healthy and diseased fields to identify those most strongly associated with BRR. Factors significantly correlated with BRR symptoms were soil compaction, fine soil texture, absence of raised beds, high application rates of the herbicide terbacil, advanced age of planting, nonuse of the fungicide metalaxyl, and cumulative years of strawberry monoculture. Populations of Pratylenchus spp. were not associated with poor root health. The data suggest that most factors that compromise root growth may predispose strawberry plants to infection by site-specific BRR pathogens. Chemical names used: 3-tert-butyl-5-chloro-6-methyluracil (terbacil); $N$-(2,6-dimethylphenyl)- $N$ -
\end{abstract} (methoxyacetyl) alanine methyl ester (metalaxyl).

Blackening of roots with associated poor growth and yield is common among strawberry plantings worldwide. In many cases, the causal agent of this blackening is unknown, and the disease symptoms are referred to as black root rot (BRR) (Wilhelm, 1984). Most studies on the etiology of BRR have approached the problem by seeking to identify the pathogen(s) present in diseased plantings. Many biotic causal factors have been implicated in the disease (Wing et al., 1994a). For example, BRR has been attributed to Rhizoctonia fragariae Husain \& McKeen in Italy (D'Ercole et al., 1989); Ontario (Husain and McKeen, 1963); Oregon (Zeller, 1932); Connecticut (Martin, 1988); California (Wilhelm et al., 1972); Massachusetts (Drozdowski,

Received for publication 14 Apr. 1994. Accepted for publication 27 Aug. 1994. Department of Fruit and Vegetable Science paper no. 49. Article is a portion of MS thesis submitted to Cornell Univ. by $\mathrm{K}$. Wing. Ian Merwin's review of the manuscript is gratefully acknowledged. We thank Judith Burr and J. Ronald Nevill for technical assistance; Peter Mullin for assistance with nematode identifications; Russell Coombe, Edward Rutkowski, Kenneth Silsby, Warren Smith, Craig Telgheder, Stephen Vandermark, and Deborah Breth for assistance in identifying study sites; and the North American Strawberry Growers Association, the New York Berry Growers Association, and Hatch Project NY142-402 for funding the project. Use of trade names does not imply endorsement of the products named nor criticism of similar ones not named. The cost of publishing this paper was defrayed in part by the payment of page charges. Under postal regulations, this paper therefore must be hereby marked advertisement solely to indicate this fact.
1987); and West Virginia, Maryland, Pennsylvania, and Arkansas (Ribeiro and Black, 1971). It also has been attributed to various Pythium spp. in Japan (Watanabe et al., 1977), Illinois (Nemec, 1970; Nemec and Sanders, 1970), and California (Wilhelm, 1965), and to Pratylenchus penetrans (Cobb) Filip. \& Stek. in the Netherlands (Klinkenberg, 1955), Ontario (Hildebrand and West, 1941; Townshend, 1962), Connecticut (LaMondia and Martin, 1989), Massachusetts (Goheen and Bailey, 1955), and New Hampshire (Chen and Rich, 1962). These putative pathogens are widespread in strawberry plantings (Wilhelm, 1984), yet BRR does not always occur where these organisms are present (Wing et al., 1994a), suggesting that one or more environmental factors may predispose strawberry plants to infection.

Although BRR is common and serious in New York, its causal agents are unknown; consequently, no generally effective controls for this complex disease have been identified. The objective of this work was to identify environmental and cultural factors that warrant further emphasis in studies on predisposition, causality, and disease control of BRR using a comprehensive field survey of cultural and soil factors associated with BRR.

\section{Materials and Methods}

New York field survey. Environmental data were collected in May and June 1992 because symptoms are most apparent shortly before and during the fruiting stage (personal observation). Furthermore, Spring 1992 was cooler and wetter than normal throughout the state, conditions reported to be favorable for BRR development (Wilhelm, 1984). In Ithaca, for example, March through May precipitation was $240 \mathrm{~mm}$ (30 $\mathrm{mm}$ above average) and the mean temperature was $5 \mathrm{C}$ (1C below average). The sample population was comprised of 27 farms selected from all major strawberry growing regions of New York (number of farms): Finger Lakes (three), Great Lakes (eight), Southern Tier (five), Adirondacks (three), Long Island (three), and Hudson Valley (five). Fields were selected on the recommendation of local extension agents and were representative of diverse northeastern United States growing conditions. At each farm, data were collected at two locations: one where plants were most vigorous, and one where plants were declining and showing symptoms typically attributed to BRR.

Growers were interviewed for data on cultural practices and field histories. We obtained information on chemicals used and their rates, cultivars, age of planting, cover crops used, previous cropping history, and irrigation and mulching practices.

Field measurements. A 1- to 5-point scale was used to rate the health of feeder and perennial roots of at least five plants at a location where plants were healthy, and another five where plants were declining. For perennial roots, 1 = entirely black; $2=$ greater than $90 \%$ black; $3=$ moderate decay; $4=$ less than $10 \%$ black; and $5=$ abundant, fleshy, and white. For feeder roots, $1=$ nonexistent; $2=$ very few; $3=$ some present; $4=$ many present; and $5=$ abundant, fleshy, and white.

The distribution of low-vigor plants within each farm was characterized as being one or more of the following: uniform across the farm; along rows; at field edges; in dry or wet areas; comprising large $\left(>1 \mathrm{~m}^{2}\right)$ or small $\left(<1 \mathrm{~m}^{2}\right)$ areas; or in stony areas. Soil strength was measured with a cone penetrometer (DickeyJohn, Auburn, Ill.) at depths of 10, 18, and 26 $\mathrm{cm}$ at two or more places within each site and then averaged.

Weed species at each location were identified and classified as to family. The occurrence of plant families also was correlated with root scores.

Laboratory analyses. Soil nutrient concentrations, $\mathrm{pH}$, and organic matter percentage were determined by the Cornell Nutrient Analysis Laboratory (sodium acetate extraction). To determine the sand, silt, and clay composition of soils, the hydrometer method of particle-size analysis was used (Day, 1965). Samples for nematode analyses from each paired site at each farm consisted of five fullroot systems and 20 bulked soil cores (Barker, 1985). Soil population densities were estimated by counting nematodes extracted from $100 \mathrm{~cm}^{3}$ of soil by sucrose flotation and subsequent centrifugation; root population densities were estimated from fresh roots shaken for $24 \mathrm{~h}$ in water and sieved (Niblack and Hussey, 1985). Nematodes were identified to genus (Mai and Lyon, 1975). Ten plants from each site were dried, and average weights for shoots and roots were determined. 
Statistical analysis. Simple regressions were performed between measured variables and root scores, and $P$ values were obtained to measure the strength of relationships. Neither feeder root scores nor perennial root scores were obviously superior as a predictive measure of overall plant vigor as reflected in total plant dry weight (correlations of 0.52 and 0.53 , respectively), so both were used as response variables in separate simple regressions (Table 1).

Some independent variables were binary in nature. By convention, 1 indicates that the location was positive for the particular variable and 0 indicates that the location did not exhibit the characteristic. The mean of a binary variable indicates the proportion of the sampled population that exhibits the characteristic. When a variable is not binary, the mean indicates the average value for the sampled population. Also reported are the coefficients of determination adjusted for degrees of freedom ( ) to indicate the proportion of the variation in feeder root scores explained by regression. The sign of the regression coefficient also is given where $P<$ 0.10 (Table 1). Similar statistics are included for the simple regressions of each factor on perennial root scores. Variables with a linear association with root scores were ranked by their $P$ value.

\section{Results and Discussion}

Many variables were measured at 27 farms, but several had probable relationships with the initiation of BRR disease. No single factor appeared to represent a substantial part of the observed variation in root health scores, as indicated by the low values (Table 1). This finding may reflect the diversity of soil environments sampled and the nonspecificity of symptoms scored, and suggests that BRR may be caused by different factors in different fields or that several interacting factors were necessary to cause disease. Thus, our results are consistent with Wilhelm's (1984) characterization of BRR as a disease complex. However, some factors were significantly correlated with both perennial and feeder root health at or above the $90 \%$ confidence level. These factors may help identify conditions that favor BRR, even though they may not be sufficient to cause disease.

A few variables were highly correlated with perennial- but not feeder-root scores (or vice versa); these may be spurious relationships. Alternatively, perennial root damage might be fundamentally different from feederroot damage. The longer life span of perennial roots may expose them to a greater number of predisposing factors, or the lack of lignification in feeder roots might make them more vulnerable to infection by pathogens. Since both feeder-root and perennial-root damage appear to be important in the stunting and decline of plants affected by BRR, each is considered here.

Physical and chemical soil factors. Better root health was associated with samples from dry or sandy areas within the field, while poor root health was associated with compaction in the root zone and high soil clay and silt content (Table 1). These results are consistent with observations of others that BRR commonly occurs in poorly drained, clayey, compacted soils (Lipman, 1914; Nemec, 1970; Watanabe et al., 1977). Cultural practices or site selection criteria favoring adequate internal drainage and soil aeration might reduce the probability that BRR will develop.

BRR was associated with fields having high levels of soil $\mathrm{Mg}$, low levels of soil $\mathrm{Al}$, and where preplant soil tests indicated that the soil supply of $\mathrm{K}$ might be deficient $(<170$ $\left.\mathrm{kg} \cdot \mathrm{ha}^{-1}\right)$. However, none of the soil chemical factors appeared to play a major role in predisposing plants to BRR.

Cultural factors. Flat beds $(<10 \mathrm{~cm}$ above row aisles) were associated with poor root health, whereas beds raised 10 to $20 \mathrm{~cm}$ were associated with better root health. None of the growers interviewed created raised beds intentionally, but beds can become higher than aisles when soil is thrown over row centers during renovation. Elevation of beds may reduce the incidence or severity of disease by decreasing soil water matric potential, increasing soil $\mathrm{O}_{2}$ concentration, and decreasing the length of time soils are saturated with water in the root zone. Soil saturation can cause physiological injury to strawberry roots, and favors the activity of some pathogens, such as Pythium and Phytophthora, that produce zoospores requiring free water for motility (Galletta and Himelrick, 1990; Griffin, 1963). Although each of these cultural or soil factors can compromise root health directly, our data suggest that plants exhibit BRR when soil pathogens are present under these conditions.

Three factors related to the history of strawberry production in the sampled fields were significantly associated with poor root health: age of the current planting, strawberry production on the site within the previous 5 years, and cumulative period of strawberry production. These associations suggest that an increase in pathogen populations with prolonged strawberry culture might be important in the development of BRR. Other researchers have noted the gradual onset of disease and suggested that a buildup of pathogen populations over several years appeared to be correlated with the severity of strawberry root decay (Berkeley and Lauder-Thomson, 1934; Hildebrand and West, 1941; Zeller 1932). Continuous strawberry production also can negatively influence soil structure and chemistry, and contribute to the accumulation of herbicide residues.

Three pesticides were associated with root scores: terbacil herbicide, metalaxyl fungicide, and fumigation. Higher application rates of the herbicide terbacil (Sinbar; E.I. du Pont de Nemours and Co., Wilmington, Del.) were associated with poor root health. Numerous studies have shown that terbacil applied at the rate of $0.56 \mathrm{~kg} \cdot \mathrm{ha}^{-1}$ can reduce vigor of strawberries, but none investigated a relationship between root health and terbacil use (Ahrens, 1982; Jensen, 1979; Langer, 1973; Masiunas and Weller, 1986; Poling and Monaco, 1985; Uprichard, 1972; Weller, 1981, 1984). High levels of terbacil may stress plants, predisposing them to BRR pathogens. This association was not found for the other six herbicides used on strawberries.

Use of the fungicide metalaxyl (Ridomil; CIBA-GEIGY, Greensboro, N.C.) was associated with better root health. Metalaxyl is a narrow-spectrum fungicide, effective primarily against oomycetes, such as Pythium spp., but not against most other fungi implicated in BRR. Wing et al. (1994b) and Watanabe et al. (1977) reported that Pythium spp. are a major component of BRR when soil temperatures are low, which may explain why metalaxyl use is positively correlated with root health in New York where soils are generally cool. Metalaxyl cannot control BRR where fungi other than oomycetes have a role. Thus, selective use of fungicides may be a tool for characterizing the principal pathogens involved in specific BRR episodes.

Use of a soil fumigant was correlated with poor root health in our study. It may be that fumigation increases the severity of disease by a "boomerang effect"; i.e., the dominant pathogen eventually reappears in greater quantities after fumigation because populations of competing microorganisms have been reduced by fumigation (Altman, 1970; Kreutzer, 1965). An alternative interpretation of this correlation is that fields with a previous history of root disease were more likely to have been fumigated. Fumigation is an expensive procedure that is used by less than half of the growers surveyed in New York (Table 1). In contrast to the farms in our survey, fumigation has effectively controlled BRR in several other locations (Klinkenberg, 1955; Raski, 1956; Wilhelm, 1965; Wolfe et al., 1990). Although fumigation may be done thoroughly, its effectiveness could be negated by using transplants that are already infected with BRR or are carrying inoculum on their roots.

The cultivar Blomidon had healthier roots than 'Earliglow'. However, field trials in New York and Massachusetts in which strawberry cultivars were scored for BRR resistance have produced inconsistent rankings (Cooley et al., 1993; Wing, 1994; Wing et al., 1994b). Thus, the relative susceptibility of strawberry cultivars to BRR is uncertain, butrelative susceptibility could vary with location and cause of the disease.

Nematodes.Pratylenchus spp. populations in soil samples were positively related to root health, and the association between root scores and Pratylenchus counts from root samples was not significant. This contrasts with the finding of others that higher root-lesion nematode (P. penetrans) populations are associated with more severe BRR (Goheen and Smith, 1956; Hildebrand and West, 1941; Klinkenberg, 1955; LaMondia and Martin, 1989; Townshend, 1962, 1963). Our correlation could be a reflection of sampling technique, as nematodes will migrate out of decaying roots. However, BRR can occur where nematodes are not found (Wing, 1994; Wing et al., 1994b), and soil conditions most strongly associated with BRR (heavy, wet, compacted soils) are those that least favor nematodes. Therefore, the role of nematodes in BRR is unclear. 
Table 1. Descriptive statistics and significance of simple regression coefficients for variables measured in a 1992 New York field survey of factors associated with strawberry black root rot. For binary variables, 1 indicates a positive score for the variable, and 0 indicates a negative score.

\begin{tabular}{|c|c|c|c|c|c|c|c|c|c|c|}
\hline \multirow[b]{2}{*}{ Variables } & \multicolumn{4}{|c|}{ Descriptive statistics } & \multicolumn{3}{|c|}{$\begin{array}{l}\text { Regression against } \\
\text { feeder-root score }\end{array}$} & \multicolumn{3}{|c|}{$\begin{array}{c}\text { Regression against } \\
\text { perennial-root score }\end{array}$} \\
\hline & Mean & SD & Min & Max & $\begin{array}{c}P \\
\text { value }^{\mathrm{x}}\end{array}$ & w & $\begin{array}{c}\text { Coefficient } \\
\text { sign }^{v}\end{array}$ & $\begin{array}{c}P \\
\text { value }\end{array}$ & & $\begin{array}{c}\text { Coefficient } \\
\text { sign } \\
\end{array}$ \\
\hline \multicolumn{11}{|l|}{ Physical $^{\mathrm{u}}$} \\
\hline Low-vigor plants occur in dry areas $(0 / 1)^{t}$ & 0.08 & 0.3 & 0 & 1 & $<0.01$ & 0.21 & + & $<0.01$ & 0.15 & + \\
\hline 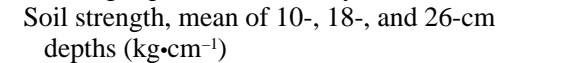 & 11 & 4 & 3 & 17 & 0.01 & 0.18 & - & 0.01 & 0.16 & - \\
\hline Low-vigor plants occur in areas $>1 \mathrm{~m}^{2}(0 / 1)$ & 0.33 & 0.5 & 0 & 1 & 0.01 & 0.13 & + & 0.03 & 0.08 & + \\
\hline Soil texture: sand (\%) & 49 & 18 & 15 & 88 & 0.02 & 0.10 & + & 0.19 & 0.02 & \\
\hline Soil texture: clay (\%) & 20 & 7 & 4 & 37 & 0.03 & 0.07 & - & 0.10 & 0.04 & - \\
\hline Soil texture: silt (\%) & 30 & 13 & 7 & 55 & 0.04 & 0.07 & - & 0.38 & 0.00 & \\
\hline Low-vigor plants occur in stony areas $(0 / 1)$ & 0.04 & 0.2 & 0 & 1 & 0.15 & 0.02 & & 0.20 & 0.02 & \\
\hline \multicolumn{11}{|l|}{ Soil coarse fraction: $0=$ no stones, to } \\
\hline $6=$ very gravelly & 2 & 2 & 0 & 6 & 0.16 & 0.03 & & 0.31 & 0.00 & \\
\hline Low-vigor plants occur along rows $(0 / 1)$ & 0.13 & 0.3 & 0 & 1 & 0.18 & 0.02 & & 0.03 & 0.08 & - \\
\hline Low-vigor plants occur in areas $<1 \mathrm{~m}^{2}(0 / 1)$ & 0.29 & 0.5 & 0 & 1 & 0.20 & 0.02 & & 0.27 & 0.01 & \\
\hline Low-vigor plants occur uniformly & & & & & & & & & & \\
\hline throughout field $(0 / 1)$ & 0.33 & 0.5 & 0 & 1 & 0.31 & 0.00 & & 0.33 & 0.00 & \\
\hline Low-vigor plants occur at field edges $(0 / 1)$ & 0.04 & 0.2 & 0 & 1 & 0.50 & 0.00 & & 0.53 & 0.00 & \\
\hline Soil temperature at $150-\mathrm{cm}$ depth $\left({ }^{\circ} \mathrm{C}\right)$ & 16 & 2 & 12 & 21 & 0.51 & 0.00 & & 0.47 & 0.00 & \\
\hline Low-vigor plants occur in low areas $(0 / 1)$ & 0.21 & 0.4 & 0 & 1 & 0.69 & 0.00 & & 0.99 & 0.00 & \\
\hline Low-vigor plants occur in wet areas $(0 / 1)$ & 0.08 & 0.3 & 0 & 1 & 0.91 & 0.00 & & 0.99 & 0.00 & \\
\hline \multicolumn{11}{|l|}{ Soil chemistry } \\
\hline $\mathrm{Zn}\left(\mathrm{kg} \cdot \mathrm{ha}^{-1}\right)$ & 5 & 11 & 1 & 67 & 0.11 & 0.03 & & 0.22 & 0.01 & \\
\hline $\mathrm{Mg}\left(\mathrm{kg} \cdot \mathrm{ha}^{-1}\right)$ & 370 & 190 & 44 & 850 & 0.15 & 0.02 & & 0.06 & 0.05 & - \\
\hline $\mathrm{Al}\left({\left.\mathrm{kg} \cdot h \mathrm{~h}^{-1}\right)}^{1}\right.$ & 77 & 45 & 15 & 213 & 0.16 & 0.02 & & 0.10 & 0.04 & + \\
\hline $\mathrm{K}$ below recommended level of 170 & & & & & & & & & & \\
\hline$\left(\mathrm{kg} \cdot \mathrm{ha}^{-1}\right)(0 / 1)$ & 0.1 & 0.3 & 0.0 & 1.0 & 0.28 & 0.00 & & 0.02 & 0.10 & - \\
\hline $\mathrm{Fe}\left(\mathrm{kg} \cdot \mathrm{ha}^{-1}\right)$ & 11 & 6 & 3 & 39 & 0.45 & 0.00 & & 0.43 & 0.00 & \\
\hline $\mathrm{P}\left(\mathrm{kg} \cdot \mathrm{ha}^{-1}\right)$ & 46 & 31 & 6 & 131 & 0.55 & 0.00 & & 0.46 & 0.00 & \\
\hline $\mathrm{NO}_{3}-\mathrm{N}\left(\mathrm{kg} \cdot \mathrm{ha}^{-1}\right)$ & 21 & 33 & 8 & 223 & 0.59 & 0.00 & & 0.24 & 0.01 & \\
\hline Organic matter (percent by weight) & 3.4 & 1.5 & 0.7 & 7.2 & 0.62 & 0.00 & & 0.75 & 0.00 & \\
\hline Exchangable acidity (meq/100 g) & 3.2 & 4.4 & 0.0 & 14.3 & 0.82 & 0.00 & & 0.71 & 0.00 & \\
\hline Conductivity $(\mathrm{mmho} / \mathrm{cm})$ & 0.1 & 0.1 & 0.1 & 0.5 & 0.85 & 0.00 & & 0.66 & 0.00 & \\
\hline $\mathrm{pH}$ & 6.3 & 0.5 & 5.2 & 7.5 & 0.88 & 0.00 & & 0.95 & 0.00 & \\
\hline $\mathrm{Ca}\left(\mathrm{kg}_{\mathrm{hh}}{ }^{-1}\right)$ & 2,500 & 1,900 & 400 & 12,500 & 0.89 & 0.00 & & 0.52 & 0.00 & \\
\hline $\operatorname{Mn}\left(\mathrm{kg} \cdot \mathrm{ha}^{-1}\right)$ & 35 & 34 & 4 & 230 & 0.98 & 0.00 & & 0.99 & 0.00 & \\
\hline $\mathrm{K}\left(\mathrm{kg} \cdot \mathrm{ha}^{-1}\right)$ & 370 & 140 & 120 & 730 & 0.98 & 0.00 & & 0.48 & 0.00 & \\
\hline \multicolumn{11}{|l|}{ Cultural } \\
\hline Beds not raised $(<10 \mathrm{~cm}$ above row aisle $)(0 / 1)$ & 0.72 & 0.5 & 0 & 1 & $<0.01$ & 0.18 & - & 0.05 & 0.06 & - \\
\hline Herbicide: terbacil application rate ( $\mathrm{kg}$ a.i./ha) & 0.40 & 0.3 & 0 & 1 & $<0.01$ & 0.26 & - & 0.10 & 0.05 & - \\
\hline Beds raised 10 to $20 \mathrm{~cm}$ above aisle $(0 / 1)$ & 0.16 & 0.4 & 0 & 1 & $<0.01$ & 0.16 & + & 0.03 & 0.08 & + \\
\hline Planting age (years elapsed since establishment) & 2.6 & 1.3 & 1 & 5 & $<0.01$ & 0.15 & - & 0.03 & 0.08 & - \\
\hline Fungicide: metalaxyl applied $(0 / 1)$ & 0.34 & 0.5 & 0 & 1 & 0.01 & 0.16 & + & 0.07 & 0.06 & + \\
\hline Cultivar: Blomidon $(0 / 1)$ & 0.06 & 0.2 & 0 & 1 & 0.01 & 0.12 & + & 0.06 & 0.05 & + \\
\hline Cumulative years of strawberry production & 7 & 6 & 1 & 30 & 0.01 & 0.12 & - & 0.04 & 0.07 & - \\
\hline Field never subsoiled (e.g., chisel plowed) $(0 / 1)$ & 0.48 & 0.5 & 0 & 1 & 0.02 & 0.10 & - & 0.44 & 0.00 & \\
\hline Legume grown within last 5 years $(0 / 1)$ & 0.09 & 0.3 & 0 & 1 & 0.03 & 0.09 & + & 0.18 & 0.02 & \\
\hline Herbicide: terbacil applied $(0 / 1)$ & 0.77 & 0.4 & 0 & 1 & 0.04 & 0.07 & - & 0.16 & 0.02 & \\
\hline Fungicide: captan applied (0/1) & 0.61 & 0.5 & 0 & 1 & 0.04 & 0.08 & - & 0.22 & 0.01 & \\
\hline Field subsoiled at renovation only $(0 / 1)$ & 0.48 & 0.5 & 0 & 1 & 0.05 & 0.07 & + & 0.45 & 0.00 & \\
\hline Strawberries grown within last 5 years $(0 / 1)$ & 0.59 & 0.5 & 0 & 1 & 0.06 & 0.06 & - & 0.09 & 0.05 & - \\
\hline Field fumigated before planting $(0 / 1)$ & 0.45 & 0.5 & 0 & 1 & 0.08 & 0.05 & - & 0.02 & 0.11 & - \\
\hline Corn grown within last 5 years $(0 / 1)$ & 0.36 & 0.5 & 0 & 1 & 0.08 & 0.05 & + & 0.46 & 0.00 & \\
\hline Cultivar: Earliglow $(0 / 1)$ & 0.31 & 0.5 & 0 & 1 & 0.09 & 0.04 & - & 0.09 & 0.04 & - \\
\hline Cultivar: Allstar $(0 / 1)$ & 0.15 & 0.4 & 0 & 1 & 0.11 & 0.04 & & 0.13 & 0.03 & \\
\hline Herbicide: sethoxydim applied $(0 / 1)$ & 0.11 & 0.3 & 0 & 1 & 0.13 & 0.03 & & 0.26 & 0.01 & \\
\hline Cultivar: Jewel $(0 / 1)$ & 0.06 & 0.2 & 0 & 1 & 0.13 & 0.03 & & 0.63 & 0.00 & \\
\hline Cultivar: Lateglow $(0 / 1)$ & 0.06 & 0.2 & 0 & 1 & 0.14 & 0.03 & & 0.39 & 0.00 & \\
\hline Alfalfa grown within last 5 years $(0 / 1)$ & 0.04 & 0.2 & 0 & 1 & 0.14 & 0.03 & & 0.28 & 0.00 & \\
\hline Herbicide: $2,4-\mathrm{D}$ application rate (kg a.i./ha) & 0.27 & 0.5 & 0 & 2 & 0.14 & 0.04 & & 0.16 & 0.03 & \\
\hline Area in strawberry production (ha) & 4 & 3 & 0.4 & 8 & 0.14 & 0.04 & & 0.33 & 0.00 & \\
\hline Herbicide: $2,4-\mathrm{D}$ applied $(0 / 1)$ & 0.40 & 0.5 & 0 & 1 & 0.14 & 0.03 & & 0.08 & 0.05 & - \\
\hline Herbicide: glyphosate applied $(0 / 1)$ & 0.19 & 0.4 & 0 & 1 & 0.19 & 0.02 & & 0.23 & 0.01 & \\
\hline Herbicide: napropamide application rate & & & & & & & & & & \\
\hline (kg a.i./ha) & 1.6 & 2.9 & 0 & 5 & 0.19 & 0.02 & & 0.88 & 0.00 & \\
\hline Row spacing: $150-\mathrm{cm}$ row centers $(0 / 1)$ & 0.08 & 0.3 & 0 & 1 & 0.19 & 0.02 & & 0.11 & 0.04 & \\
\hline Fungicide: benomyl applied (0/1) & 0.49 & 0.5 & 0 & 1 & 0.21 & 0.02 & & 0.49 & 0.00 & \\
\hline Plant source: Nourse Farms nursery (0/1) & 0.52 & 0.5 & 0 & 1 & 0.21 & 0.01 & & 0.27 & 0.01 & \\
\hline Small grain grown within last 5 years $(0 / 1)$ & 0.20 & 0.4 & 0 & 1 & 0.26 & 0.01 & & 0.40 & 0.00 & \\
\hline Raspberries grown within last 5 years $(0 / 1)$ & 0.07 & 0.3 & 0 & 1 & 0.27 & 0.01 & & 0.99 & 0.00 & \\
\hline Plant source: Aherns nursery $(0 / 1)$ & 0.02 & 0.1 & 0 & 1 & 0.28 & 0.01 & & 0.54 & 0.00 & \\
\hline Herbicide: DCPA applied (0/1) & 0.09 & 0.3 & 0 & 1 & 0.29 & 0.00 & & 0.10 & 0.04 & \\
\hline
\end{tabular}




\begin{tabular}{|c|c|c|c|c|c|c|c|c|c|c|}
\hline \multirow[b]{2}{*}{ Variables } & \multicolumn{4}{|c|}{ Descriptive statistics } & \multicolumn{3}{|c|}{$\begin{array}{l}\text { Regression against } \\
\text { feeder-root score }\end{array}$} & \multicolumn{3}{|c|}{$\begin{array}{l}\text { Regression against } \\
\text { perennial-root score }^{y}\end{array}$} \\
\hline & Mean & SD & Min & $\operatorname{Max}$ & $\begin{array}{c}P \\
\text { value }^{\mathrm{x}} \\
\end{array}$ & w & $\begin{array}{c}\text { Coefficient } \\
\text { sign }\end{array}$ & $\begin{array}{c}P \\
\text { value }\end{array}$ & & $\begin{array}{c}\text { Coefficient } \\
\text { sign } \\
\end{array}$ \\
\hline Row spacing: >150-cm row centers (0/1) & 0.04 & 0.2 & 0 & 1 & 0.29 & 0.00 & & 0.32 & 0.00 & \\
\hline Irrigation: $1=$ overhead, $0=$ no irrigation & 0.96 & 0.2 & 0 & 1 & 0.29 & 0.00 & & 0.32 & 0.00 & \\
\hline Herbicide: napropamide applied $(0 / 1)$ & 0.57 & 0.5 & 0 & 1 & 0.30 & 0.00 & & 0.78 & 0.00 & \\
\hline Beds raised $>20 \mathrm{~cm}$ above row aisle $(0 / 1)$ & 0.12 & 0.3 & 0 & 1 & 0.32 & 0.00 & & 0.82 & 0.00 & \\
\hline Solanceous crop grown within last 5 years $(0 / 1)$ & 0.13 & 0.3 & 0 & 1 & 0.37 & 0.00 & & 0.59 & 0.00 & \\
\hline Row spacing: $120-\mathrm{cm}$ row centers $(0 / 1)$ & 0.46 & 0.5 & 0 & 1 & 0.38 & 0.00 & & 0.87 & 0.00 & \\
\hline Irrigation water recirculated $(0 / 1)$ & 0.15 & 0.4 & 0 & 1 & 0.39 & 0.00 & & 0.45 & 0.00 & \\
\hline Cultivar: Raritan $(0 / 1)$ & 0.06 & 0.2 & 0 & 1 & 0.42 & 0.00 & & 0.04 & 0.07 & + \\
\hline Plant source: Norcal nursery $(0 / 1)$ & 0.04 & 0.2 & 0 & 1 & 0.43 & 0.00 & & 0.94 & 0.00 & \\
\hline Field subsoiled at renovation and planting $(0 / 1)$ & 0.04 & 0.2 & 0 & 1 & 0.45 & 0.00 & & 0.98 & 0.00 & \\
\hline \multicolumn{11}{|l|}{ Fumigant: methyl isocyanate/ } \\
\hline dichloropropanes $(0 / 1)$ & 0.27 & 0.5 & 0 & 1 & 0.47 & 0.00 & & 0.31 & 0.00 & \\
\hline Hay grown within last 5 years $(0 / 1)$ & 0.16 & 0.4 & 0 & 1 & 0.53 & 0.00 & & 0.49 & 0.00 & \\
\hline Fungicide: thiophanate-methyl applied $(0 / 1)$ & 0.10 & 0.3 & 0 & 1 & 0.59 & 0.00 & & 0.35 & 0.00 & \\
\hline Fungicide: thiram applied $(0 / 1)$ & 0.07 & 0.3 & 0 & 1 & 0.60 & 0.00 & & 0.90 & 0.00 & \\
\hline \multicolumn{11}{|l|}{ Herbicide not labeled for use in New York } \\
\hline applied $(0 / 1)$ & 0.09 & 0.3 & 0 & 1 & 0.65 & 0.00 & & 0.36 & 0.00 & \\
\hline Fungicide: vinclozolin applied $(0 / 1)$ & 0.66 & 0.5 & 0 & 1 & 0.66 & 0.00 & & 0.14 & 0.03 & \\
\hline Fumigant: methyl dithiocarbamate applied (0/1) & 0.04 & 0.2 & 0 & 1 & 0.67 & 0.00 & & 0.34 & 0.00 & \\
\hline Fungicide: iprodione applied $(0 / 1)$ & 0.10 & 0.3 & 0 & 1 & 0.75 & 0.00 & & 0.82 & 0.00 & \\
\hline Cultivar: Honeyoe $(0 / 1)$ & 0.19 & 0.4 & 0 & 1 & 0.77 & 0.00 & & 0.66 & 0.00 & \\
\hline Tree fruit grown within last 5 years $(0 / 1)$ & 0.04 & 0.2 & 0 & 1 & 0.78 & 0.00 & & 0.84 & 0.00 & \\
\hline Row spacing: <120-cm row centers $(0 / 1)$ & 0.42 & 0.5 & 0 & 1 & 0.80 & 0.00 & & 0.51 & 0.00 & \\
\hline Herbicide: paraquat applied $(0 / 1)$ & 0.04 & 0.2 & 0 & 1 & 0.87 & 0.00 & & 0.72 & 0.00 & \\
\hline Plant source: Brittingham's nursery $(0 / 1)$ & 0.11 & 0.3 & 0 & 1 & 0.88 & 0.00 & & 0.82 & 0.00 & \\
\hline Rows $>30 \mathrm{~cm}$ wide $(0 / 1)$ & 0.86 & 0.4 & 0 & 1 & 0.90 & 0.00 & & 0.90 & 0.00 & \\
\hline Rows $<30 \mathrm{~cm}$ wide $(0 / 1)$ & 0.14 & 0.4 & 0 & 1 & 0.90 & 0.00 & & 0.90 & 0.00 & \\
\hline Curcubits grown within last 5 years $(0 / 1)$ & 0.11 & 0.3 & 0 & 1 & 0.90 & 0.00 & & 0.93 & 0.00 & \\
\hline Brassicas grown within last 5 years $(0 / 1)$ & 0.09 & 0.3 & 0 & 1 & 0.98 & 0.00 & & 0.60 & 0.00 & \\
\hline Root pruning at planting $(0 / 1)$ & 0.15 & 0.4 & 0 & 1 & 0.99 & 0.00 & & 0.63 & 0.00 & \\
\hline \multicolumn{11}{|l|}{ Nematodes } \\
\hline Pratylenchus (no./100 cm³ soil) & 23 & 44 & 0 & 240 & 0.05 & 0.06 & + & 0.10 & 0.04 & + \\
\hline Paratylenchus (no./g fresh root) & 0 & 2 & 0 & 13 & 0.12 & 0.03 & & 0.36 & 0.00 & \\
\hline Meloidogyne (no./g fresh root) & 0 & 0 & 0 & 3 & 0.19 & 0.02 & & 0.52 & 0.00 & \\
\hline Aphelenchoides (no./g fresh root) & 1 & 4 & 0 & 22 & 0.22 & 0.01 & & 0.30 & 0.00 & \\
\hline Paratylenchus (no./100 $\mathrm{cm}^{3}$ soil) & 19 & 54 & 0 & 331 & 0.47 & 0.00 & & 0.80 & 0.00 & \\
\hline Pratylenchus (no./g fresh root) & 31 & 115 & 0 & 784 & 0.64 & 0.00 & & 0.64 & 0.00 & \\
\hline Meloidogyne (no./100 $\mathrm{cm}^{3}$ soil) & 7 & 26 & 0 & 146 & 0.65 & 0.00 & & 0.54 & 0.00 & \\
\hline Aphelenchoides (no./100 cm³ soil) & 6 & 14 & 0 & 60 & 0.93 & 0.00 & & 0.08 & 0.05 & + \\
\hline \multicolumn{11}{|l|}{ Plant response [dry wt $(\mathrm{g})]$} \\
\hline Whole plant & 12.4 & 6.2 & 2.4 & 29.3 & $<0.01$ & 0.26 & - & $<0.01$ & 0.27 & - \\
\hline Shoot & 7.0 & 4.4 & 0.9 & 19.1 & $<0.01$ & 0.27 & - & $<0.01$ & 0.30 & - \\
\hline Root & 5.5 & 2.6 & 1.5 & 11.3 & 0.02 & 0.10 & - & 0.03 & 0.08 & - \\
\hline
\end{tabular}

${ }^{\mathrm{u}}$ Feeder roots were rated on a 1 to 5 scale, where $1=$ roots nonexistent and $5=$ roots abundant, fleshy, and white.

yerennial roots were rated on a 1 to 5 scale, where $1=$ roots entirely black and $5=$ roots abundant, fleshy, and white.

${ }^{x}$ Significance level of the estimated simple regression coefficient for individual variables.

w $=$ Coefficient of determination, adjusted for degrees of freedom.

${ }^{v}$ Coefficient sign $=$ sign of estimated simple regression coefficient from regression of each variable against root scores; not shown unless $P$ value for either feederor perennial-root score $<0.10$.

"Variables ranked within each category by $P$ value of feeder-root score simple regression coefficient.

${ }^{\mathrm{t}} 0 / 1=$ coded as binary variable.

Interpretation of correlations. Ideally, multivariate statistics would be used to analyze the BRR survey data. However, multivariate techniques require larger sample sizes than we could collect, given time and financial constraints. Univariate statistics were the best alternative, despite a number of limitations. Simple regression ignores interactions between variables - effects that are not currently understood but are potentially important in a disease as complex as BRR. Moreover, when the factors themselves are not independent, the strength of relationships indicated by simple regression may be overestimated or underestimated. For instance, bed height increased with planting age in the fields examined, so the true benefit of raised beds may have been somewhat masked in the analysis by the deleterious effect of continuous strawberry production.

Several groups of variables that we measured were intercorrelated, making the identification of single predisposing factors impossible. An example is soil strength, clay content, and soil K concentration. Separate experiments would be required to quantify the effects of individual variables on BRR. Although one would expect certain correlations to exist within locations (e.g., age of planting and bed height, or age of planting and cumulative years in strawberries), these were not significant across our heterogeneous sample.

In summary, this preliminary study documents some of the edaphic and cultural factors that may influence the severity of BRR in New York. Sites with good soil physical properties and a short history of strawberry production favored healthy roots and were associated with an absence of BRR. Raised beds, the use of metalaxyl fungicide, and nonuse of terbacil were associated positively with root health. The value of fumigation for the control of BRR appeared to be questionable, and broad resistance to the BRR disease complex did not appear to exist in the major cultivars.

Future research on the etiology and control of BRR should examine the associations between root decay and soil physical properties, and the population dynamics of purported pathogens under continuous strawberry production at individual sites. Strawberry production on raised beds in nonfumigated soil without terbacil herbicide should be compared to conventional practices in several sites that appear to favor the development of BRR. 


\section{Literature Cited}

Ahrens, J.F. 1982. Napropamide and terbacil for newly planted strawberries. Adv. Strawberry Prod. 1:22-26.

Altman, J. 1970. Increased and decreased plant growth responses resulting from soil fumigation, p. 216-221. In: T.A. Toussoun, R.V. Bega, and P.E. Nelson (eds.). Root diseases and soil borne pathogens. Univ. of California Press, Berkeley.

Barker, K.R. 1985. Soil sampling methods and procedures for field diagnosis, p. 11-20. In: B.F. Zuckerman, W.F. Mai, and M.B. Harrison (eds.). Plant nematology laboratory manual. Univ. of Massachusetts Agr. Expt. Sta., Amherst.

Berkeley, G.H. and I. Lauder-Thomson. 1934. Root rots of strawberry in Britain, the "black lesion" type of strawberry root rot. Pomol. Hort. Sci. 12:222-246.

Chen, T. and A.E. Rich. 1962. The role of Pratylenchus penetrans in the development of strawberry black root rot. Plant Dis. Rptr. 46:839 843.

Cooley, D.R., A.F. Tuttle, and S.G. Schloemann. 1993. Integrated management of black root rot of strawberry; an update of variety tolerance research. Northeast SARE Small Fruits Nwsl. 4(1):11-16. Dept. of Plant Pathology, Univ. of Massachusetts, Amherst.

Day, P.R. 1965. Particle fractionation, p. 562-566. In: C.A. Black (ed.). Methods of soil analysis, Part I. Amer. Soc. Agron., Madison, Wis.

D’Ercole, N., P. Nipoti, and D. Manzali. 1989. Research on the root rot complex of strawberry plants. Acta Hort. 265:497-506.

Drozdowski, J. 1987. A survey of fungi associated with strawberry black root rot in commercial fields. Adv. Strawberry Prod. 6:47-49.

Galletta, G.J. and D.G. Himelrick (eds.). 1990. Small fruit crop management. Prentice-Hall, Englewood Cliffs, N.J.

Goheen, A.C. and J.S. Bailey. 1955. Meadow nematodes in strawberry plantings in Massachusetts. Plant Dis. Rptr. 39:879-880.

Goheen, A.C. and J.B. Smith. 1956. Effects of inoculation of strawberry roots with meadow nematodes, Pratylenchus penetrans. Plant Dis. Rptr. 40:146-149.

Griffin, D.M. 1963. Soil physical factors and the ecology of fungi. II. Behavior of Pythium ultimum at small soil water suctions. Trans. Brit. Mycol. Soc. 46:368-372.

Hildebrand, A.A. and P.M. West. 1941. Strawberry root rot in relation to microbial changes induced in root rot soil by the incorporation of certain cover crops. Can. J. Res. 19c(6).

Husain, S.S. and W.E. McKeen. 1963. Rhizoctonia fragariae sp. nov. in relation to strawberry degeneration in southwestern Ontario. Phytopathology 53:532-540.

Jensen, K.I.N. 1979. Herbicide programs with terbacil in first-year strawberries. Weed Abstr. 28:131.

Klinkenberg, C.H. 1955. Nematode diseases of strawberries in the Netherlands. Plant Dis. Rptr. 39:603-606.

Kreutzer, W.A. 1965. The reinfestation of treated soil, p. 495-508. In: K.F. Baker and W.C. Snyder (eds.). Ecology of soil-borne plant pathogens. Univ. of California Press, Berkeley.

LaMondia, J.A. and S.B. Martin. 1989. The influence of Pratylenchus penetrans and temperature on black root rot of strawberry by binucleate Rhizoctonia spp. Plant Dis. 73:107-110.

Langer, C.A. 1973. The use of pronamide and terbacil in strawberry weed control. Northeastern Weed Soc. Proc. 27:252-256.

Lipman, C.B. 1914. The management of strawberry soils in the Pajaro Valley and its problems. California Agr. Expt. Sta. Circ. 122.

Mai, W.F. and H.H. Lyon. 1975. Pictorial key to genera of plant-parasitic nematodes. Cornell Univ. Press, Ithaca, N.Y.

Martin, S.B. 1988. Identification, isolation frequency and pathogenicity of anastomosis groups of binucleate Rhizoctonia spp. from strawberry roots. Phytopathology 78:379-384.

Masiunas, J.B. and S.C. Weller. 1986. Strawberry cultivar response to postplant applications of terbacil. HortScience 21:1147-1149.

Nemec, S. 1970. Pythium sylvaticum - Pathogenic on strawberry roots. Plant Dis. Rptr. 54:416418.

Nemec, S. and H. Sanders. 1970. Pythium species associated with strawberry root necrosis in southern Illinois. Plant Dis. Rptr. 54:49-51.

Niblack, T.L. and R.S. Hussey. 1985. Extracting nematodes from soil and plant tissue, p. 201206. In: B.F. Zuckerman, W.F. Mai, and M.B. Harrison (eds.). Plant nematology laboratory manual. Univ. of Massachusetts Agr. Expt. Sta., Amherst.

Poling, E.B. and J.J. Monaco. 1985. Activated charcoal root dips enhance herbicide selectivity in strawberries. HortScience 20:251-252.

Raski, D.J. 1956. Pratylenchus penetrans tested on strawberries grown in black root rot soil. Plant Dis. Rptr. 40:690-693.

Ribeiro, O.K. and L.L. Black. 1971. Rhizoctonia fragariae: A mycorrhizal and pathogenic fungus of strawberry plants. Plant Dis. Rptr. 55:599603.

Townshend, J.L. 1962. The root-lesion nematode, Pratylenchus penetrans (Cobb, 1917) Filip. and Stek. 1941, in strawberry in the Niagara Peninsula and Norfolk County in Ontario. Can. J. Plant Sci. 42:728-736.

Townshend, J.L. 1963. The pathogenicity of Pratylenchus penetrans to strawberry. Can. J. Plant Sci. 43:75-78.

Uprichard, S.D. 1972. Response of newly planted 'Cambridge Vigour' strawberries to pronamide, terbacil and bromacil. Proc. 11th Brit. Weed Control Conf. p. 395-397.

Watanabe, T., K. Hashimoto, and M. Sato. 1977. Pythium species associated with strawberry roots in Japan and their role in strawberry stunt disease. Phytopathology 67:1324-1332.

Weller, S.C. 1981. Response of strawberry cultivars to post-planting applications of terbacil and napropamide. Weed Sci. Soc. Amer. Mtg. Abstr. 33.

Weller, S.C. 1984. Evaluation of postplant applications of terbacil and napropamide to strawberry plants. Adv. Strawberry Prod. 3:15-19.

Wilhelm, S. 1965. Pythium ultimum and the soil fumigation growth response. Phytopathology 55:1015-1020.

Wilhelm, S. 1984. Black root rot, p. 90. In: J.L. Maas (ed.). Compendium of strawberry diseases. APS Press, St. Paul, Minn.

Wilhelm, S., P.E. Nelson, H.E. Thomas, and H. Johnson. 1972. Pathology of strawberry root rot caused by Ceratobasidium species. Phytopathology 62:700-705.

Wing, K.B. 1994. The etiology of strawberry black root rot disease. MS Thesis, Cornell Univ., Ithaca, N.Y.

Wing, K.B., M.P. Pritts, and W.F. Wilcox. 1994a. Strawberry black root rot. Adv. Strawberry Res. 13:13-19.

Wing, K.B., M.P. Pritts, and W.F. Wilcox. 1994b. Field resistance of 20 strawberry cultivars to black root rot. Fruit Var. J. (In press.)

Wolfe, D., J.R. Hartman, G.R. Brown, and J. Strang. 1990. The influence of soil fumigation on strawberry yield and economics in black root rot infested fields. Applied Agr. Res. 5:17-20.

Yuen, G.Y., M.N. Schroth, A.R. Weinhold, and J.G. Hancock. 1991. Effects of soil fumigation with methyl bromide and chloropicrin on root health and yield of strawberry. Plant Dis. 75:416-420.

Zeller, S.M. 1932. A strawberry disease caused by Rhizoctonia. Oregon Agr. Expt. Sta. Bul. 295. 